\title{
How to Secure a Wireless Sensor Network
}

\author{
Yee Wei Law \\ Paul J.M. Havinga \\ Faculty of Electrical Engineering, Mathematics and Computer Science \\ University of Twente, PO Box 217, 75000 AE Enschede, The Netherlands \\ \{yee.wei.law, paul.havinga\}@utwente.nl
}

\begin{abstract}
The security of wireless sensor networks (WSNs) is a complex issue. While security research of WSNs is progressing at a tremendous pace, and many security techniques have been proposed, no comprehensive framework has so far emerged that attempts to tie the bits and pieces together to ease the implementors' adoption of the technologies. We answer the challenge by proposing a guidelines according to which WSN security can be implemented in practice.
\end{abstract}

\section{INTRODUCTION}

Security allows WSNs to be used with confidence. Without security, the use of WSN is any application domain would result in undesirable consequences. Table 1 serves as an illustration of what might happen to WSNs in the absence of security, categorized according to application domains. However, the implementation of security itself remains much of an art today. Although much progress has been made for the past few years, the field remains fragmented, with contributions dispersed over seemingly disjoint yet actually connected areas, for example key management only makes sure the communicating nodes (one source node and one sink node for ease of discussion) possess the necessary keys, at the same time protecting the confidentiality, integrity and authenticity of the communicated data. To guarantee the sink node receives the data at all within a certain time limit, secure multipath routing is required. Key management and secure multipath routing are but two components of the bigger puzzle that is WSN security.

Our contribution is therefore to provide a step-by-step, layer-by-layer guide to securing a WSN using existing technologies. We do so by picking up the individual puzzles littered over the security research landscape and put them together in a concise and comprehensive framework. Emerging from the framework is a set of guidelines that offer a systematic view of state-of-the-art WSN security. More importantly, our guidelines should make WSN implementators find security more approachable. We know of no existing work that attempts to perform the organization that we have done.

The rest of this paper is organized as follows. Section 2 lays down the principles on top of which our guidelines are built. Section 3 gives the first part of our guidelines from a host-based perspective. Section 4 then presents the second part of our guidelines from a network-based perspective. Section 5 concludes.

\section{Principles}

Security mechanisms are devised based on a set of principles. Among these principles, some are common security strategies, some are direct consequences of the hardware restrictions of sensor nodes. The principles are described here to pave way for the guidelines in the next section:

1) Energy efficiency: The requirement for energy efficiency suggests that in most cases computation is favored over communication, as communication is three orders of magnitude more expensive than computation [1]. The requirement also suggests that security should never be overdone - on the contrary, tolerance is generally preferred to overaggressive prevention [2].

2) No public-key cryptography: Public-key algorithms remain prohibitively expensive on sensor nodes both in terms of storage and energy [3]. No security schemes should rely on public-key cryptography.

3) No tamper-resistance: Since sensor nodes are low-cost hardware that are not built with tamper-resistance in mind, their strength has to lie in their number - a few 'fallen' nodes should not bring the entire network down. The network should instead be resilient to attacks. The concept of resilience, or equivalently, redundancy-based defense is widely cited $[2,4,5]$.

4) Multiple layers of defense: The fact that attacks can occur on different layers of a networking stack (as defined in the Open System Interconnect model) suggests that there should be multiple layers of defense, i.e. a separate defense for each layer [2].

These principles are independent of the specific traits of the application domain, so they are generally applicable.

\section{Host-Based Defense Guidelines}

The guidelines are built bottom-up. As with wired networks, an attack on WSNs might start with compromising a node and spread to other nodes. Therefore, we first consider the sensor nodes themselves as standalone devices (host-based defense), then we take communications into account (network-based defense) in the next section.

Since physical tampering cannot be avoided, care must at least be taken to prevent software tampering in the form of malicious code (malcode). Chances are the operating system and/or the applications running in a sensor node are vulnerable to popular exploits such as buffer overflows. There are more 
Table 1: Potential security threats, Grouped aCCORding to application domains. SA=SERVice AVAilability, C=Confidentiality, I=INTEGRITY, A=AUTHENTICITY.

\begin{tabular}{|c|c|c|c|c|c|}
\hline \multirow{2}{*}{$\begin{array}{l}\text { Application } \\
\text { domain }\end{array}$} & \multirow{2}{*}{ Potential security threats } & \multicolumn{4}{|c|}{ Properties violated } \\
\hline & & SA & $\mathrm{C}$ & $\mathrm{I}$ & $\mathrm{A}$ \\
\hline Military & $\begin{array}{l}\bullet \text { Denial-of-service attacks by means of jamming and/or confusing the networking protocols. } \\
\bullet \text { Eavesdropping of classified information. } \\
\bullet \text { Supply of misleading information, e.g. enemy movements in the East where in fact they are in the West. }\end{array}$ & $x$ & $x$ & $\bar{x}$ & $\times$ \\
\hline $\begin{array}{l}\text { Disaster detection } \\
\text { and relief }\end{array}$ & $\begin{array}{l}\text { - Supply of misleading information, e.g. bogus disaster warnings, by pranksters, causing huge financial loss as a } \\
\text { result of unnecessary large-scale evacuation and deployment of relief equipments. }\end{array}$ & & & & $x$ \\
\hline Industry & $\begin{array}{l}\bullet \text { Eavesdropping of commercial secrets by business rivals. } \\
\bullet \text { Intentional disruption of manufacturing processes as a result of misleading sensor readings caused by disgruntled } \\
\text { employees or business spies. }\end{array}$ & $x$ & $x$ & $x$ & \\
\hline Agriculture & $\begin{array}{l}\text { - The agricultural department might want to deploy WSNs to ensure that farmers do not overuse pesticides or other } \\
\text { hazardous chemicals on their crops, but unscrupulous farmers might tamper with the sensor nodes. }\end{array}$ & & & $\bar{x}$ & \\
\hline $\begin{array}{l}\text { Environmental } \\
\text { monitoring }\end{array}$ & $\begin{array}{l}\text { - Suppose government-endorsed environmental sensors are installed near a factory to monitor air/water quality to } \\
\text { make sure the factory's emission lies beneath the pollution threshold, however by feeding the sensors with wrong } \\
\text { information, the factory allows itself to escape detection and let its polluting emission go unchecked. }\end{array}$ & & & $x$ & \\
\hline $\begin{array}{l}\text { Intelligent } \\
\text { buildings }\end{array}$ & $\begin{array}{l}\text { - Biometrics-based access control mechanisms are compromisable if the biometric sensors can be bypassed or } \\
\text { fooled. } \\
\text { - Token-based access control mechnisms are compromisable if the token authentication protocol is insecure. }\end{array}$ & $\times$ & & $x$ & $\times$ \\
\hline Health/medical & $\begin{array}{l}\text { Providing wrong physiological measurements of a patient to the carer or doctor, a miscreant may cause potentially } \\
\text { fatal diagnosis and treatment to be performed on the patient. }\end{array}$ & & & $\times$ & $x$ \\
\hline Law enforcement & $\begin{array}{l}\text { - If criminals are able to eavesdrop the databases of the police departments, or to misguide the detection of gunshots, } \\
\text { or to disrupt the network, public safety will be affected. }\end{array}$ & $x$ & $\times$ & $x$ & $x$ \\
\hline Transportation & - There is no order in the city when traffic information can no longer be trusted because they can easily be spoofed. & & & $\times$ & $x$ \\
\hline Space exploration & $\begin{array}{l}\text { - Space agencies invest billions into space exploration projects, it is only logical they want to ensure all commands } \\
\text { executed on their space probes are authorized, and all collected data encrypted and authenticated. }\end{array}$ & $\times$ & $\times$ & $\times$ & $\times$ \\
\hline
\end{tabular}

risks for operating systems that support dynamic loading of code module over the network [6]. Writing secure code is fortunately an established discipline. There is a vast body of literature on the subject [7]. There are also existing and mature tools for spotting memory errors in programs, so writing secure programs is a non-issue. Mobile code is however problematic. Code-signing, using symmetric keys per Principle 2, alone is insufficient in identifying malicious mobile code since the owner of the signing key might have been compromised. Although techniques are being developed to facilitate the safe execution of untrusted code [8], the overhead involved is nontrivial. The recommendation is thus: mobile code should only be enabled when strictly necessary, per Principle 1, in which case all mobile code should be signed and should carry a proof. The key used for signing the code is determined by the underlying key management scheme, which will be discussed later.

\section{NETWORK-BASED DEFENSE GUIDELINES}

An attack coming from the network to a sensor node is either aimed at disrupting the operation of the sensor node or getting information from the sensor node. Hence we are mainly looking at two types of protection: protection from denial-of-service (DoS) attacks, and protection of the secrecy of information. Multiple defenses, each for one layer of the networking stack should be implemented, per Principle 4. One layer is discussed at a time:

\section{A. Physical Layer}

The physical layer defines the actual methods used to transmit and receive information through the radio interface, for example the frequency, the data rate, the signal modulation and the spread spectrum scheme to use. DoS attacks on the physical layer are radio jamming. Well-known countermeasures to radio jamming include adaptive antenna systems, spread spectrum modulations, error correcting codes and cryptography. There is not much room to maneuver in antenna systems and error correcting codes because sensor nodes typically use an omnidirectional antenna and Reed-Solomon codes [9-12]. Sectored antennas as suggested by Noubir [13] are not yet available for sensor nodes. We concentrate on spread spectrum modulations in this section, and will talk about cryptography in Section 4E.

Ideally the transceiver should support some form of spread spectrum modulation, preferrably frequency-hopping spread spectrum (FHSS), instead of direct-sequence spread spectrum (DSSS). FHSS is preferred to DSSS, because DSSS requires more circuitry (higher cost) to implement, is more energyconsuming and more sensitive to environmental effects [14, 15]; on the other hand, the hop rate in a FHSS system is typically much lower than the chip rate in a DSSS system, resulting in lower energy usage $[14,16]$. According to Table 2, only one of the most common sensor node transceivers supports DSSS, and among the rest, half of the transceivers support FHSS and the other half do not support spread spectrum at all. We do not recommend DSSS transceivers.

TABLE 2: COMPARISON OF SPREAD SPECTRUM SUPPORT.

\begin{tabular}{|l|l|}
\hline Spread spectrum & Transceiver \\
\hline DSSS & Chipcon CC2400 [17] \\
\hline FHSS & $\begin{array}{l}\text { Chipcon CC1000 [18], Conexant Systems RDSSS9M [15], } \\
\text { MaxStream 9XStream [19], Zeevo Bluetooth [20] }\end{array}$ \\
\hline No & $\begin{array}{l}\text { Infineon TDA 5250 [21], National Semiconductor } \\
\text { LMX3162 [22], RFM TR1000 [23], RFM TR1001 [24] }\end{array}$ \\
\hline
\end{tabular}

For FHSS transceivers, we recommend quaternary/binary frequency-shift keying (FSK) as the data modulation scheme 
of choice $[14,25]$, and a hop rate between 500 and 1000 hops/s [26]. A maximum hop rate of 1000 hops/s does not hamper the most sophisticated interceptors [12], but per Principle 1, 500-1000 hops/s should be a practical compromise. The exact hop rate should be picked by taking into account the fact that lower hop rate gives larger transmission range, but higher hop rate gives lower response time [16].

For transceivers that do not support spread spectrum, we recommend the channel surfing method by $\mathrm{Xu}$ et al [27]. Channel surfing is essentially an adaptive form of frequency hopping. Instead of continuously hopping from frequency to frequency, a node only switches to a different, orthogonal frequency $\pm \delta$ away when it discovers the current frequency is being jammed. The value of $\delta$ can be determined by experiments, e.g. for Berkeley motes, $\delta$ is found to be multiples of $800 \mathrm{kHz}$ [27]. A node can determine if the medium is being jammed if the packet delivery ratio is low but the signal strength of its neighbor is high [28].

\section{B. Data Link Layer}

The data link layer defines how data are encoded and decoded, how errors are detected and corrected, the addressing scheme as well as the medium access scheme. The anti-jam strategies in the previous section are mostly effective against 'dumb' jammers. According to our latest results in link-layer jamming [29,30], smart jammers can take advantage of the data link layer to achieve energy-efficient jamming. In the earlier work [29], we show that S-MAC can be jammed energy-efficiently by jamming the control interval of the listen interval alone [29], so we recommend encrypting packets on the data link layer, for example as done in TinySec [31]. An elaborate encryption scheme depends on the key management architecture which will be discussed in Section 4-E. In the latter work [30], we show that even when the packets are encrypted, the temporal arrangement of the packets induced by the nature of the protocol unravel patterns that the jammer can take advantage of. The significance of this result is that to the attackers, compared to radio jamming, link-layer jamming offers better energy efficiency, and compared to DoS attacks on the upper layers, link-layer jamming requires less implementation and no need to know the content of the packets. According to our analysis [30], in the absence of effective countermeasures, TDMA protocols like LMAC [32] have better anti-jam properties, and therefore should be preferred to other protocols like S-MAC [33,34] and B-MAC [35].

\section{Network Layer}

The network layer is responsible for routing messages from one node to another node multiple hops away, based on some system-defined parameters such as energy or latency. There are 2 types of routing protocols for WSNs: (1) ID-based protocols, in which packets are routed to the destination designated by the ID specified in the packets themselves; and (2) data-centric protocols [36], in which packets contain attributes that specify what kinds of data are being requested or provided. The literature considers any action that results in any combination of the following an attack [37]:

1) Packets are dropped completely, or selectively forwarded.

2) The network is flooded with global broadcasts.

3) Some sensor nodes in the network are misguided into believing that nodes that either are multiple hops away, or that do not exist at all are their neighbors. This is called a Sybil attack.

4) A significant proportion of the traffic is tunneled from one place in the network to another distant place of the network, depriving other parts of the network that under normal circumstances would have received the traffic themselves. This is called a wormhole attack.

5) A significant proportion of the traffic is lured to a particular node or a small group of nodes, depriving other parts of the network that under normal circumstances would have received the traffic themselves. This is called a sinkhole or black hole attack. This attack can be facilitated by the wormhole attack.

6) Some routes form loops or detours.

These attacks are sophisticated forms of DoS attacks. Among these attacks, we ignore the last one because we do not see any significant value for the attackers in it - causing loops is not more efficient than just dropping packets; causing detours is an inefficient way of wasting the sensor nodes' energy.

The first attack is countered using multipath routing [38].

The second attack is countered using authenticated broadcasts, which has to be facilitated by the underlying key management architecture.

Sybil, wormhole and sinkhole attacks require the attacker to forge packets. To prevent this, a key management architecture is required. In particular, Sybil attacks can be countered using random key pre-distribution schemes [39], to be discussed in Section 4-E.

Against wormhole attacks and hence sinkhole attacks, so far there is no resource-lean and energy-efficient countermeasure [40-42], i.e. with or without key management, wormhole and sinkhole attacks are still an open issue [37].

We now describe our recommendation. Consistent with Karlof et al.'s analysis [37], we recommend using datacentric protocols such as multipath directed diffusion [38], or geographic routing protocols $[43,44]$ in case the nodes are able to determine their own locations, because these protocols include flooding as a robust way of disseminating information. The security of geographic routing protocols depends on the correctness of the location information, as such secure geographic routing requires secure localization, which is discussed in the next section. In conjunction with these protocols, the data link layer should support encryption and authentication, just as we have recommended in the previous section, whereas the key management architecture should support authenticated broadcasts and random key pre-distribution. In general, the above strategy is not effective against wormhole and sinkhole attacks but we believe that the data link layer is easier to DoSattack than the network layer, so if the security of the network 
cannot be helped, the data link layer should at least be made as resistant to DoS attacks as possible.

If there is a need to use ID-based routing, we recommend endairA [45], an improved version of Ariadne [46], because it is provably secure against an attacker with a single compromised key and a single compromised node. However, it has to be extended to support multipath routing. Furthermore, the corresponding key management architecture has to support node-specific key pre-distribution (i.e. every node has to share one key with every other node in the network), in addition to authenticated broadcasts.

Note that per Principle 2, we do not consider public-keybased secure routing protocols at all [47-50].

\section{Application Layer}

The application layer manages data. What is most important is the correctness of the collected data. It is common for data to be aggregated, for example, the temperature readings of a particular region of the network to be averaged. However averaging is not a secure aggregation function [51]. A better solution is to use the median of the data. An aggregation function can be, and should always be determined to whether or not be resilient to attacks using Wagner's technique [51]. However it is important to note that Wagner's result is only applicable if the aggregator node is in range with all the source nodes, that is if there's no other intervening aggregator between the aggregator and the source nodes. This scheme is applicable to cluster-based networks where a clusterhead can act as an aggregator for its cluster members.

The communication channel between an aggregator and a home server that is operated by a human user has potentially limited bandwidth, because an aggregator might be a sensor node. To guarantee that if the home server accepts an aggregation result from the aggregator, the reported result is close to the true aggregation value with high probability, Przydatek et al. [52] propose a communication-efficient transaction paradigm called aggregate-commit-prove, which in effect provides two layers of defense against data corruption. The first defense is commitment (hence the word 'commit' in aggregate-commit-prove): the aggregator commits to the aggregated data, by cryptographic means. The second defense is interactive proofs (hence the word 'prove'): the aggregator proves to the base station the validity of the aggregation result, by statistical means. The aggregator and home server need to share a key with each of the source nodes.

We consider secure localization as an application service, as location is a form of data. As mentioned in the previous section, secure geographic routing depends on secure localization. Lazos et al.'s secure localization scheme [53] works on the assumptions that (1) the locators, i.e. the devices that provide trusted location information to other nodes, are tamper-resistant, and (2) the density of locators is known to every node.

\section{E. Cross-Layer}

There are two cross-layer services that are of concern: (1) key management, and (2) intrusion detection and response.
Key management is the process by which cryptographic keys are generated, stored, protected, transferred, loaded, used, and destroyed. At the outset, per Principle 2 and 3, schemes like the pebblenets [54], the terminodes $[55,56]$ and Luo et al.'s [57] are ruled out. The key management scheme we are recommending is based on Zhu et al.'s LEAP [58], extended according to our earlier analysis of the data link layer, the network layer and the application layer. Before giving the details, it is useful to discuss an important concept of LEAP: passive participation. In passive participation, a node that overhears its neighbor's transmitted data may choose not to transmit its data if its neighbor's data are the same as its own data, thereby saving energy. To support passive participation, a node has to share a key, called by Zhu et al. the cluster key, with its neighbors. The node also has to establish a one-way key chain [59] and send the commitment of the key chain to its neighbors to allow its neighbors to authenticate its locally broadcast messages. The combination of a cluster key and a one-way key chain is interesting because

- if only the cluster key is used, a compromised neighbor would disclose the cluster key;

- if only the key chain is used, the keys in the key chain would have to be broadcast in the clear, allowing replay attacks to take place;

- but if used together, the cluster key can be used to hide the keys in the key chain from cluster-outsiders, so that the keys do not need to be disclosed according to a schedule as in SPINS, and the keys in the key chain can be used for authentication as usual.

We now present the details. The network can be bootstrapped, and nodes can be added/removed following the protocols of LEAP [58]. We deduce the following keys are needed on each sensor node (for ease of discussion, we give the role of sink nodes / aggregators / locators to the base stations):

- For link-layer encryption and authentication, 1 network-wide key.

- For authenticated broadcasts, $b$ commitments of $b$ oneway key chains, where $b$ is the number of base stations that are authorized to perform global broadcasts.

- If the routing protocol is a data-centric protocol and the network is static, $d$ pairwise keys that are shared with the node's $d$ neighbors.

If the routing protocol is a data-centric protocol and the network is mobile, a fixed number $k$ of randomly pre-distributed keys.

Note: these $d$ pairwise keys, or $k$ randomly pre-distributed keys are for countering Sybil attacks. Using $k$ randomly pre-distributed keys allows a node to be mobile and yet establish secure connections with new neighbors, although with reduced connectivity.

If the routing protocol is endairA (the only ID-based routing protocol we recommend), $n-1$ keys where $n$ is the total number of nodes in the network.

- For Przydatek et al.'s secure information aggrega- 
tion [52] (as discussed in the previous section), 1 shared key with the base station and 1 shared key with the home server if the node is a source node.

- For passive participation, $d+1$ cluster keys, $d$ commitments of the one-way key chains of its neighbors, 1 one-way key chain of itself, where $d$ is the number of neighbors.

An energy-efficient key management scheme is built on top of energy-efficient cryptographic primitives, namely block ciphers, message authentication codes (MACs) and hash functions (public-key crypto is excluded per Principle 2). Among these primitives, we are only concerned with block ciphers, because MACs can be constructed from block ciphers [60], and hash functions are relatively cheap [61]. Based on our extensive investigation of the security properties as well as storage- and energy-efficiency of a few carefully selected ciphers [62,63], our recommendation is as shown in Figure 1. The recommended operation mode is OFB for pairwise links, but $\mathrm{CBC}$ for group communications.

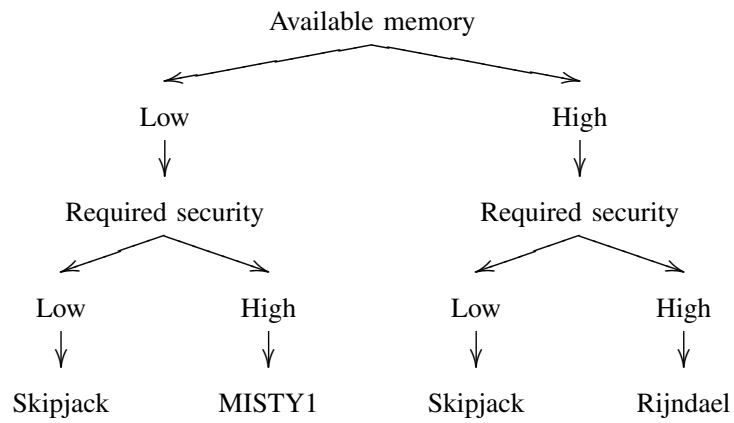

Fig. 1: Selection of an energy-efficient cipher under the constraints of available memory and required security.

Intrusion detection and response is the process of monitoring anomalies on the network, collecting evidence on whether the anomalies are a result of intrusions and responding with appropriate countermeasures. What should be classified as an anomaly depends strongly on the application. A sensor node can recognize some anomalies as intrusions on its own, e.g. whether it is jammed (Section 4-A), but for some other anomalies like whether it is being targeted with Sybil attacks, it needs to collect evidence from its neighbors (Section 4-C). In view of the possibility that some of the neighbors themsevles might be malicious, several reputation-based schemes have been proposed [64-67]. In these schemes, a node rates the reputation of a neighbor based on whether the neighbor is 'cooperative'. A node's ability to evaluate cooperativeness is however questionable. For example, the fact that a node receive data from its neighbor does not imply the neighbor is cooperating if the data is false. The node might consult other neighbors on the validity of the data, but depending on what value is measured, the other neighbors may or may not be able to assure the node of the data's validity, moreover instead of requiring a node to constantly monitor its neighbors' transmitted data, which is energy-consuming [68], it makes more economic sense from an energy perspective to delegate the role to a secure aggregator (Section 4-D). Therefore per Principle 1, we do not see reputation-based schemes as playing an essential role in the security of WSNs. Different intrusion responses are applicable to different layers, and are already discussed in the respective layers.

\section{Conclusion}

Practical WSN security is a balancing act that is constantly in search of the highest level of protection that can be squeezed out of the judicious use of limited resources. We have achieved the objective of putting together the individual puzzles that comprise WSN security in the form of a comprehensive set of guidelines. However an integrated design approach should yield better results than what we have done here. For example, we are looking for better integrated secure routing and key management architecture. Until then, our guidelines should serve the need of the time.

\section{REFERENCES}

[1] A. S. Wander, N. Gura, H. Eberle, V. Gupta, and S. C. Shantz, "Energy analysis of public-key cryptography for wireless sensor networks," in Third IEEE International Conference on Pervasive Computing and Communications (PERCOM'05). IEEE Computer Society Press, 2005, pp. 324-328.

[2] H. Yang, H. Luo, F. Ye, S. Lu, and L. Zhang, "Security in mobile ad hoc networks: challenges and solutions," IEEE Wireless Communications, vol. 11, no. 1, pp. 38-47, Feb. 2004.

[3] D. Carman, B. Matt, D. Balenson, and P. Kruus, "A communications security architecture and cryptographic mechanisms for distributed sensor networks," in DARPA SensIT Workshop. NAI Labs, The Security Research Division Network Associates, Inc., 1999. [Online]. Available: http://download.nai.com/products/media/pgp/pdf/ sensit-workshop-100799.pdf

[4] H. Chan, A. Perrig, and D. Song, "Random key predistribution schemes for sensor networks," in Proceedings of the 2003 IEEE Symposium on Security and Privacy. IEEE Computer Society, 2003.

[5] A. Perrig, J. Stankovic, and D. Wagner, "Security in wireless sensor networks," Commun. ACM, vol. 47, no. 6, pp. 53-57, 2004.

[6] T. Hofmeijer, S. Dulman, P. Jansen, and P. Havinga, "AmbientRT real time system software support for data centric sensor networks," in Proceedings of the Intelligent Sensors, Sensor Networks and Information Processing Conference. IEEE, 2004, pp. 61-66.

[7] M. G. Graff and K. R. V. Wyk, Secure Coding: Principles and Practices. O'Reilly, 2003.

[8] G. C. Necula, "Proof-carrying code (abstract): design, implementation and applications," in PPDP 'OO: Proceedings of the 2nd ACM SIGPLAN international conference on Principles and practice of declarative programming. New York, NY, USA: ACM Press, 2000, pp. 175-177.

[9] G. Lin and G. Noubir, "Low Power DOS Attacks in Data Wireless LANs and Countermeasures," Northeastern University, Tech. Rep., 2002. [Online]. Available: http://www.ccs.neu.edu/home/noubir/ publications/LN02a.pdf

[10] _ "On Link Layer Denial of Service in DATA Wireless LANs," Wiley Journal on Wireless Communications and Mobile Computing, To appear. [Online]. Available: http://www.ccs.neu.edu/home/noubir/ publications/LN05.pdf

[11] G. Noubir and G. Lin, "Low-power DoS attacks in data wireless LANs and countermeasures," SIGMOBILE Mob. Comput. Commun. Rev., vol. 7, no. 3, pp. 29-30, 2003.

[12] D. C. Schleher, Electronic Warfare in the Information Age. Artech House, July 1999.

[13] G. Noubir, "On connectivity in ad hoc networks under jamming using directional antennas and mobility," in Wired/Wireless Internet Communications, ser. LNCS, vol. 2957. Springer-Verlag, 2004, pp. 186-200.

[14] J. Min, "Analysis and design of a frequency-hopped spread-spectrum transceiver for wireless personal communications," Ph.D. dissertation, University of California, Los Angeles, 1995. [Online]. Available: http://www.icsl.ucla.edu/aagroup/PDF_files/tcvr-arch.pdf 
[15] G. J. Pottie and L. P. Clare, "Wireless integrated network sensors: toward low-cost and robust self-organizing security networks," in Sensors, C3I, Information, and Training Technologies for Law Enforcement, ser. SPIE Proceedings, vol. 3577, 1999, pp. 86-95. [Online]. Available: http://wins.rsc.rockwell.com/publications/spie3577-12.pdf

[16] K. Tovmark, Chipcon Application Note AN014: Frequency Hopping Systems (Rev. 1.0), Chipcon AS, Mar. 2002. [Online]. Available: http //www.chipcon.com/files/AN_014_Frequency_Hopping_Systems_1_0.pdf

[17] SmartRF ${ }^{\circledR}$ CC2420 Datasheet (rev. 1.2), Chipcon AS, June 2004 [Online]. Available: http://www.chipcon.com/files/CC2420_Data_Sheet 1_2.pdf

[18] SmartRF ${ }^{\circledR}$ CC1000 Datasheet (rev. 2.2), Chipcon AS, Apr. 2004 [Online]. Available: http://www.chipcon.com/files/CC1000_Data_Sheet_ 2_2.pdf

[19] MaxStream, "XStream ${ }^{\mathrm{TM}}$ OEM RF Module," Product Manaul v4.2A Sept. 2004. [Online]. Available: http://www.maxstream.net/products/ xstream/module/product-manual_XStream_OEM-RF-Module.pdf

[20] R. Kling, "Intel mote: An Enhanced Sensor Network Node," in International Workshop on Advanced Sensors, Structural Health Monitoring and Smart Structures, 2003. [Online]. Available: http: //www.mita.sd.keio.ac.jp/news/workshop/proceedings/Kling.pdf

[21] Wireless Components: ASK/FSK 868MHz Wireless Transceiver TDA 5250 D2 Version 1.6, 07th ed., Infineon Technologies AG, July 2002. [Online]. Available: http://www.infineon.com/cmc_upload/documents/ 046/676/TDA5250_V1.6.pdf

[22] LMX3162 Single-Chip Radio Transceiver, National Semicondutor, Mar 2000. [Online]. Available: http://cache.national.com/ds/LM/LMX3162. pdf

[23] RF Monolithics, Inc., “TR1000: 916.50 MHz Hybrid Transceiver," Datasheet, 2002. [Online]. Available: http://www.rfm.com/products/ data/tr1000.pdf

[24] — , “TR1001: 868.35 MHz Hybrid Transceiver," Datasheet, 2002. [Online]. Available: http://www.rfm.com/products/data/tr1001.pdf

[25] S. Pollin, B. Bougard, R. Mangharam, L. V. der Perre, F. Catthoor, R. Rajkumar, and I. Moerman, "Optimizing transmission and shutdown for energy-efficient packet scheduling in sensor networks," in Proceedings of the Second European Workshop on Wireless Sensor Networks (EWSN 2005). IEEE, Feb. 2005, pp. 290-301.

[26] D. J. Torrieri, "Fundamental limitations on repeater jamming of frequency-hopping communications," IEEE Journal on Selected Areas in Communications, vol. 7, no. 4, pp. 569-575, May 1989.

[27] W. Xu, T. Wood, W. Trappe, and Y. Zhang, "Channel surfing and spatial retreats: defenses against wireless denial of service," in WiSe '04: Proceedings of the 2004 ACM workshop on Wireless security. New York, NY, USA: ACM Press, 2004, pp. 80-89.

[28] W. Xu, W. Trappe, Y. Zhang, and T. Wood, "The feasibility of launching and detecting jamming attacks in wireless networks," in ACM MobiHoc '05. ACM Press, 2005, p. To appear.

[29] Y. Law, P. Hartel, J. den Hartog, and P. Havinga, "Link-layer jamming attacks on S-MAC," in 2nd European Workshop on Wireless Sensor Networks (EWSN 2005). IEEE, 2005, pp. 217-225. [Online]. Available: http://ieeexplore.ieee.org/iel5/9875/31391/01462013.pdf

[30] Y. Law, L. van Hoesel, J. Doumen, P. Hartel, and P. Havinga, "EnergyEfficient Link-Layer Jamming Attacks against Wireless Sensor Network MAC Protocols," in The Third ACM Workshop on Security of Ad Hoc and Sensor Networks (SASN 2005). ACM Press, 2005, to appear.

[31] C. Karlof, N. Sastry, and D. Wagner, "TinySec: a link layer security architecture for wireless sensor networks,' in SenSys '04: Proceedings of the 2nd international conference on Embedded networked sensor systems. New York, NY, USA: ACM Press, 2004, pp. 162-175.

[32] L. van Hoesel and P. Havinga, "A Lightweight Medium Access Protocol (LMAC) for Wireless Sensor Networks: Reducing Preamble Transmissions and Transceiver State Switches," in INSS, June 2004

[33] W. Ye, J. Heidemann, and D. Estrin, "An Energy-Efficient MAC protocol for Wireless Sensor Networks," in Proc. IEEE Infocom, USC/Information Sciences Institute. New York, NY, USA: IEEE, June 2002, pp. 1567-1576. [Online]. Available: http://www.isi.edu/ johnh/ PAPERS/Ye02a.html

[34] _ _ "Medium Access Control with Coordinated, Adaptive Sleeping for Wireless Sensor Networks," IEEE/ACM Transactions on Networking, vol. 12, no. 3, pp. 493-506, 2003. [Online]. Available: http: //ieeexplore.iee.org/iel5/90/29000/01306496.pdf

[35] J. Polastre, J. Hill, and D. Culler, "Versatile low power media access for wireless sensor networks," in SenSys '04: Proceedings of the 2nd international conference on Embedded networked sensor systems. ACM Press, 2004, pp. 95-107.

[36] D. Ganesan, A. Cerpa, Y. Yu, and D. Estrin, "Networking issues in wireless sensor networks," Journal of Parallel and Distributed Computing (JPDC), Special issue on Frontiers in Distributed Sensor Networks, To appear. [Online]. Available: http: $/ /$ lecs.cs.ucla.edu/ $\sim$ deepak/PAPERS/jpdc.pdf

[37] C. Karlof and D. Wagner, "Secure routing in wireless sensor networks: Attacks and countermeasures," Elsevier's Ad Hoc Networks Journal, Special Issue on Sensor Network Applications and Protocols, vol. 1, no. 2-3, pp. 293-315, 2003. [Online]. Available: http: //www.cs.berkeley.edu/ daw/papers/

[38] D. Ganesan, R. Govindan, S. Shenker, and D. Estrin, "Highly resilient, energy efficient multipath routing in wireless sensor networks," Mobile Computing and Communications Review (MC2R), vol. 1, no. 2, 2002.

[39] J. Newsome, E. Shi, D. Song, and A. Perrig, "The Sybil Attack in Sensor Networks: Analysis and Defenses," in IPSN 2004, Apr. 2004.

[40] Y.-C. Hu, A. Perrig, and D. Johnson, "Packet leashes: a defense against wormhole attacks in wireless networks," in 22nd Annual Joint Conference of the IEEE Computer and Communications Societies (INFOCOM 2003), vol. 3. IEEE Computer Society Press, 2003, pp. 1976-1986.

[41] S. Čapkun, L. Buttyán, and J.-P. Hubaux, "SECTOR: secure tracking of node encounters in multi-hop wireless networks," in Proceedings of the 1st ACM workshop on Security of ad hoc and sensor networks. ACM Press, 2003, pp. 21-32.

[42] W. Wang and B. Bhargava, "Visualization of wormholes in sensor networks," in Proceedings of the 2004 ACM workshop on Wireless security. ACM Press, 2004, pp. 51-60.

[43] M. Zorzi and R. R. Rao, "Geographic Random Forwarding (GeRaF) for Ad Hoc and Sensor Networks: Multihop Performance," IEEE Transactions on Mobile Computing, vol. 2, no. 4, pp. 337-348, 2003.

[44] _ , "Geographic Random Forwarding (GeRaF) for Ad Hoc and Sensor Networks: Energy and Latency Performance," IEEE Transactions on Mobile Computing, vol. 2, no. 4, pp. 349-365, 2003.

[45] L. Buttyán and I. Vajda, "Towards provable security for ad hoc routing protocols,' in SASN '04: Proceedings of the 2nd ACM workshop on Security of ad hoc and sensor networks. New York, NY, USA: ACM Press, 2004, pp. 94-105.

[46] Y.-C. Hu, A. Perrig, and D. Johnson, "Ariadne: a secure on-demand routing protocol for ad hoc networks," in Proc. 8th Annual Int. Conf. on Mobile Computing and Networking. ACM Press, 2002, pp. 12-23.

[47] L. Zhou and Z. Haas, "Securing ad hoc networks," IEEE Network, vol. 13, no. 6, pp. 24-30, 1999.

[48] S. Yi, P. Naldurg, and R. Kravets, "Security-aware ad hoc routing for wireless networks," in Proceedings of the 2001 ACM International Symposium on Mobile Ad Hoc Networking and Computing. ACM Press, 2001, pp. 299-302. [Online]. Available: http://dx.doi.org/10.1145/501449.501464

[49] M. Zapata and N. Asokan, "Securing ad hoc routing protocols," in Proceedings of the ACM workshop on Wireless security. ACM Press, 2002, pp. 1-10.

[50] K. Sanzgiri, B. Dahill, B. N. Levine, C. Shields, and E. M. BeldingRoyer, "A secure routing protocol for ad hoc networks," in Proceedings of the 10th IEEE International Conference on Network Protocols. IEEE Computer Society, 2002, pp. 78-89.

[51] D. Wagner, "Resilient aggregation in sensor networks," in Proceedings of the 2nd ACM workshop on Security of ad hoc and sensor networks. ACM Press, 2004, pp. 78-87.

[52] B. Przydatek, D. Song, and A. Perrig, "SIA: secure information aggregation in sensor networks," in Proceedings of the 1st international conference on Embedded networked sensor systems. ACM Press, 2003, pp. 255-265.

[53] L. Lazos and R. Poovendran, "SeRLoc: secure range-independent localization for wireless sensor networks," in Proceedings of the 2004 ACM workshop on Wireless security. ACM Press, 2004, pp. 21-30.

[54] S. Basagni, K. Herrin, D. Bruschi, and E. Rosti, "Secure pebblenets," in Proc. 2001 ACM Int. Symp. on Mobile Ad Hoc Networking and Computing. ACM Press, October 2001, pp. 156-163.

[55] J.-P. Hubaux, L. Buttyán, and S. Capkun, "The quest for security in mobile ad hoc networks," in Proc. of the ACM Symp. on Mobile Ad Hoc Networking and Computing (MobiHOC '01). ACM Press, October 2001, pp. 146-155.

[56] L. Buttyán and J.-P. Hubaux, "Nuglets: A Virtual Currency to Stimulate Cooperation in Self-Organized Mobile Ad Hoc Networks," 
Department of Communication Systems, Swiss Federal Institute of Technology, Tech. Rep. DSC/2001/001, 2001. [Online]. Available: http://ntrg.cs.tcd.ie/htewari/papers/tr01_001.pdf

[57] H. Luo, P. Zerfos, J. Kong, S. Lu, and L. Zhang, "Selfsecuring ad hoc wireless networks," in 7th IEEE Symp. on Computers and Communications, 2002, pp. 567-574. [Online]. Available: http://citeseer.nj.nec.com/507663.html

[58] S. Zhu, S. Setia, and S. Jajodia, "LEAP: Efficient Security Mechanisms for Large-Scale Distributed Sensor Networks," in 10th ACM Conference on Computer and Communications Security (CCS '03). ACM Press, 2003, pp. 62-72.

[59] A. Perrig, R. Szewczyk, V. Wen, D. Culler, and J. Tygar, "SPINS Security Protocols for Sensor Networks," in Proceedings of the 7th Ann. Int. Conf. on Mobile Computing and Networking. ACM Press, 2001, pp. 189-199.

[60] B. Preneel, "Cryptographic primitives for information authentication state of the art," in State of the Art in Appplied Cryptography, ser. LNCS, B. Preneel and V. Rijmen, Eds., vol. 1528. Springer-Verlag, 1998, pp. 50-105.

[61] S. Crosby and D. Wallach, "Denial of service via algorithmic complexity attacks," in 12th USENIX Security Symposium. USENIX Association, 2003, pp. 29-44. [Online]. Available: http://www.cs.rice. edu/ $\sim$ scrosby/hash/CrosbyWallach_UsenixSec2003/index.html

[62] Y. Law, J. Doumen, and P. Hartel, "Benchmarking block ciphers for wireless sensor networks (extended abstract)," in 1st IEEE International Conference on Mobile Ad-hoc and Sensor Systems (MASS 2004). IEEE Computer Society Press, Oct. 2004. [Online]. Available: http://ieeexplore.ieee.org/iel5/9587/30305/01392185.pdf

[63] - "Survey and benchmark of block ciphers for wireless sensor networks," Centre for Telematics and Information Technology, University of Twente, The Netherlands, Tech. Rep. TR-CTIT-04-07, Jan. 2004. [Online]. Available: http://www.ub.utwente.nl/webdocs/ctit/ 1/000000eb.pdf

[64] P. Michiardi and R. Molva, "Core: A COllaborative REputation mechanism to enforce node cooperation in Mobile Ad Hoc Networks," in Communications and Multimedia Security Conference, 2002. [Online]. Available: http://www.eurecom.fr/ $\sim$ michiard/pub/michiardi_adhoc_core. ps

[65] 1 , "Prevention of denial of service attacks and selfishness in mobile ad hoc networks," Institut Eurécom, France, Research Report RR-02063, 2002. [Online]. Available: http://www.eurecom.fr/ michiard/pub/ michiardi_adhoc_dos.ps

[66] - "Simulation-based analysis of security exposures in mobile ad hoc networks," in European Wireless 2002: Next Generation Wireless Networks: Technologies, Protocols, Services and Applications, February 25-28, 2002, Florence, Italy, 2002.

[67] S. Ganeriwal and M. B. Srivastava, "Reputation-based framework for high integrity sensor networks," in Proceedings of the 2nd ACM workshop on Security of ad hoc and sensor networks. ACM Press, 2004, pp. 66-77.

[68] S. Marti, T. Giuli, K. Lai, and M. Baker, "Mitigating routing misbehavior in mobile ad hoc networks," in Proc. 6th Annual Int. Conf. on Mobile Computing and Networking. ACM Press, 2000, pp. 255-265. [Online]. Available: http://citeseer.nj.nec.com/marti00mitigating.html 
